\title{
Hypoxia-induced miR-210 promoter demethylation enhances proliferation, autophagy and angiogenesis of schwannoma cells
}

\author{
ZHENGGUANG WANG ${ }^{1}$, MINGSI DENG ${ }^{2}$, ZHENDONG LIU $^{1}$ and SONG WU ${ }^{1}$ \\ ${ }^{1}$ Department of Orthopaedic Surgery, The Third Xiangya Hospital of Central South University, Changsha, Hunan 410011; \\ ${ }^{2}$ Department of Orthodontics, The Stomatological Hospital of Changsha, Changsha, Hunan 410005, P.R. China
}

Received August 17, 2016; Accepted January 4, 2017

DOI: 10.3892/or.2017.5511

\begin{abstract}
Hypoxia, a dominant feature in cancer occurrence and evolution, exists throughout the progression of most malignant tumors. This study focused on the mechanism of hypoxia-induced miR-210 upregulation, and the miR-210 functions in schwannoma. We detected microvascular density, vascular endothelial growth factor (VEGF) and miR-210 expression levels using schwannoma tissue mciroarray. The results showed that miR-210 expression was significantly associated with VEGF. Moreover, the cytological tests showed that hypoxia induced miR-210 expression, while reduce ephrin-A3 expression. The bisulfate genomic sequencing PCR results showed that miR-210 promoter region was hypermethylated in RT4-D6P2T in normoxia, while demethylated in hypoxia, and the region included the hypoxia-inducible factor- $1 \alpha$ (HIF-1 $\alpha)$ response element site. Cellular function research showed that hypoxia resulted in RT4-D6P2T apoptosis, higher autophage and invasion. Besides, hypoxia can affect HIF-1 $\alpha /$ VEGFmediated angiogenesis. To learn about the specific functions of miR-210, we found that with miR-210 inhibition, tumor cell apoptosis increased, autophagy and angiogenesis reduced, and the cell cycle was arrested. Hypoxia promoted miR-210 expression through promoter demethylation, then consequently enhanced tumor cell proliferation and autophagy, increasing tumor cell angiogenesis. Thus, miR-210 could be a potential marker for judging tumor malignancy and be taken as an effective target for clinical auxiliary treatment of neurilemmoma.
\end{abstract}

\section{Introduction}

Neurilemmoma (schwannoglioma) consists of dermatology neurofibroma and plexiform neurofibroma (PNFs), both of

Correspondence to: Dr Song Wu, Department of Orthopaedic Surgery, The Third Xiangya Hospital of Central South University, 138 Tongzipo Road, Changsha, Hunan 410011, P.R. China

E-mail: wusong2016@yeah.net

Key words: miR-210, schwannoma, methylation, apoptosis, HIF-1 $\alpha$, autophagy which are benign tumors. However, PNF has 1/10 malignant transformation rate and can evolve into malignant peripheral nerve sheath tumor (MPNST), which always metastasize and has very poor prognosis (1).

Hypoxia makes great contributions to tumor progression, oxygen supply is affected by tumor growth and following neovascularization; hypoxia can result in tumor drug resistance, metastasis and other serious consequences (2). Intracellular hypoxia can induce the HIF family to a series of responses, such as glucide metabolic pathway changes from tricarboxylic acid cycle to anaerobic glycolysis pathway, neovascularization and apoptosis $(3,4)$.

Internal hypoxic regions exist throughout growth and evolution of most malignant tumors, and such regions always necrose and are more prone to tumor metastasis (5). Hypoxia is one of cancer occurrence and evolution signs (6). The HIF-1 $\alpha$ with high expression level in the hypoxic region of tumor tissues can upregulate VEGF expression (7), and can also upregulate E-cadherin suppressor gene expression, and thus reduce inter-tumor cellular homo-adhesion and facilitate tumor cell metastasis (8). Since HIF-1 $\alpha$ hydroxylation is oxygen independent, under hypoxic conditions, HIF-1 $\alpha$ can protect itself from being degraded by proteinase von Hippel-Lindau. Then HIF-1 $\alpha$ can combine with the transcriptional activators p300, HIF-1 $\beta$ and further change its transcriptional level, and also change transcriptional levels of downstream microRNAs, including miR-210 $(3,9)$.

MicroRNAs are non-coding RNA with approximately 22 nucleotides (10). They regulate target protein expression by complete or incomplete matching with 3 ' untranslated regions (3'-UTR), which the former can directly cleave the target mRNA and further reduce target mRNA transcription and translation, and the later can repress target protein translation without affecting mRNA stability (11).

It is reported that miR-210 is associated with various tumors, such as gastroenteric tumor (12), bladder cancer (13), breast carcinoma (14), pancreatic carcinoma (15), and is also one of the major microRNAs induced by HIF-1 $\alpha$ (16). HIF-1 $\alpha$ directly combines with the hypoxia response element (HRE) at approximately 40 bp upstream of miR-210 promoter transcription start site, and cis-regulate miR-210 expression (17). miR-210 is also thought to be one of the tumor hypoxia markers (18-20).

The reported target genes of miR-210 included HOXA1, HOXA9, HOXA3, E3F3 and ephrin-A3 (EFNA3) (16), but only 
EFNA3 was still regulated by miR-210 under hypoxic conditions (21). EFNA3 is a member of Ephrins family, the biggest subtribe of receptor tyrosine kinases, of which EphA family receptors are thought to be able to inhibit tumor vasculogenesis $(22,23)$, and EphB family was thought to be associated with tumor vascular invasion and generation $(24,25)$.

Our early study results illustrated that miR-210 is associated with MPNST cell invasion and metastasis, and regulates EFNA3 protein expression via incomplete complementary paring with 3'-UTR of EFNA3, thus affects focal adhesion kinase (FAK) pathway in tumor cells and HIF-1 $\alpha /$ VEGFmediated vasculogenesis pathway $(26,27)$. However, the functions of miR-210 during neurilemmoma hypoxia, and the underlying molecular mechanism of hypoxia-induced miR-210 upregulation in schwannoma cells is still vague.

\section{Materials and methods}

Cell culture and transfections. The schwannoma RT4-D6P2T cells (ATCC, Manassas, VA, USA) were cultured in RMPI1640 (HyClone, Hudson, NH, USA) medium with $10 \%$ fetal bovine serum (Gibco, Carlsbad, CA, USA) at $37^{\circ} \mathrm{C}, 95 \%$ air and $5 \% \mathrm{CO}_{2}$ incubator (Thermo Fisher Scientific, Waltham, MA, USA), or at $37^{\circ} \mathrm{C}, 94 \% \mathrm{~N}_{2}, 5 \% \mathrm{CO}_{2}$ and $1 \% \mathrm{O}_{2}$ tri-gas incubator (Thermo Fisher Scientific) for hypoxic culture, and were subcultured every 2-3 days. HIF-1 $\alpha$-shRNApRNAT-U6.1/Neo interference plasmids (Auragene, Changsha, China), or miR-210 complementary Locked Nucleic Acid (anti-miR-210) (GeneCopopia, Rockville, MD, USA) and lipo2000 (Invitrogen Life Technologies, Carlsbad, CA, USA) mixed liquor was added into a petri dish with $30-50 \%$ cells (Nest, Wuxi, China) for 4-6 h, and then the culture medium was removed and replaced by fresh culture medium. After the replacement, the resulting solution was put in a $37^{\circ} \mathrm{C} \mathrm{CO}_{2}$ incubator for 24-72 h culture and then the cells were collected for further study.

Real-time polymerase chain reaction ( $q P C R)$. Total RNAs were extracted from the cells using TRIzol (Invitrogen Life Technologies) reagent, and prepared with a RevertAid First Strand cDNA Synthesis kit (Fermentas Inc., Ontario, Canada). SYBR green qPCR assay was carried out to find the relative expression levels of miR-210, compared with the internal control gene U6, and calculated using the $2^{-\Delta \Delta \mathrm{Ct}}$ method. The rat-miR-210 primers (RmiRQP3171), U6 primers (RmiRQP9003) and qPCR Mix were purchased from GeneCopoeia.

Western blot analysis. Cells $\left(10^{7}\right)$ were collected and put into $200 \mu \mathrm{l}$ RIPA (Invitrogen Life Technologies) lysate for extracting total proteins, of which $50 \mu \mathrm{g}$ were used for western blot analysis. The EFNA3 and $\beta$-actin antibodies were from Sigma (St. Louis, MO, USA), HIF-1 $\alpha$, P62 and elf4E antibodies were from Abcam (Cambridge, MA, USA), VEGF antibodies were from Immunoway (Suzhou, Jiangsu, China).

Cell cycle, apoptosis, migration assay, and ultrastructural observation. Cell cycle: the cells were treated for $24 \mathrm{~h}$, and then Trypsins (Auragene) were used to dissociate the cells, and PBS was used to wash and resuspend the cells. Ethyl alcohol $(70 \%)$ was added into the resuspension, then the resuspension was cultured at $4^{\circ} \mathrm{C}$ for $18 \mathrm{~h}$. Cells were again washed with PBS and the washed cells were resuspended in Staining Solution $(50 \mu \mathrm{g} / \mathrm{ml}$ of PI, $1 \mathrm{mg} / \mathrm{ml}$ of RNase A, $0.1 \%$ Triton X-100 in PBS). The stained cells $\left(1 \times 10^{6}\right)$ were then analyzed with a flow cytometer (Beckman Coulter, Brea, CA, USA). Cell apoptosis: cells $\left(5 \times 10^{5}\right)$ were resuspended in Annexin V-FITC and propidium iodide (PI) labeling solution, and then Annexin V Apoptosis Detection kit FITC (eBioscience, Affymetrix, San Diego, CA, USA) was used for immediate testing. Cell migration: Cellular invasion ability was tested by Transwell assay. The cells were cultured under normoxic/hypoxic for $24 \mathrm{~h}$, and then were dissociated and inoculated in serum-free medium in the upper chamber with well placed Matrigel for another $24 \mathrm{~h}$ in normoxic/hypoxic conditions. The remaining cells in the upper chamber were carefully removed, and the cells that entered into the Matrigel were stained by the staining solution with $75 \%$ ethyl alcohol and crystal violet (Beyotime Biotechnology, Jiangsu, China) for $25 \mathrm{~min}$. Dried at room temperature, and then were photographed with an inverted microscope (Motic, Xiamen, China). Ultrastructural observation: $24 \mathrm{~h}$ after the treatment, the cells were placed, respectively, in normoxic and hypoxic conditions for another $24 \mathrm{~h}$ culture, dissociated with TrypsinEDTA (Auragene), washed twice with PBS, immobilized with $2.5 \%$ glutaraldehyde precooled at $4^{\circ} \mathrm{C}$ overnight, post-immobilized with $2 \%$ osmic acid, dehydrated with ethanol-acetone, embedded with epoxy resin, sliced, and then observed with a transmission electron microscope (Hitachi, Tokyo, Japan) under $80 \mathrm{kV}$.

$H \& E, I H C, I S H$ and IF analysis. The schwannoma tissue microarray (SO808) was purchased from Alenabio (Shangxi, China), and hematoxylin and eosin (H\&E) staining procedures were as previously described (28). Immunology and Histology Chemistry (IHC) test: after being dewaxed, hydrated, antigen retrieved and $\mathrm{H}_{2} \mathrm{O}_{2}$ confined, the tissue chip was exposed to rabbit anti-VEGF polyclonal antibodies (1:100, Immunoway), incubated overnight at $4^{\circ} \mathrm{C}$, then exposed to goat anti-rabbit secondary antibodies (1:800, Jackson Immuno Research Inc., West Grove, PA, USA), incubated at room temperature for $30 \mathrm{~min}$, and the VEGF protein expression was observed with an optical microscope (Upototech, Changchun, China). In Situ Hybridization (ISH) test: Rat-miR-210 probe sequence: 5'-ACA GAT CAG CCG CTG TCA CAC GCA C-3', synthesized at BGI Tech (Shenzhen, China). Hybridization probe mixed solution (1:500) was instilled into the chips, using the IsHyb In Situ Hybridization kit (Biochain, Newark, CA, USA) according to the instructions, then observed and photographed the results with an optical microscope (Upototech). Immunofluorescent assay (IF) test: the cells were inoculated in a 6-well dish (the density $<80 \%$ ), after being immobilized, the anti-LC3B II/I antibodies (ab48394) by Abcam were used for IF test, with the primary antibodies diluted at a ratio of 1:1000, and the green fluorescent protein expression was observed with an inverted microscope (Motic).

Bisulfite sequencing PCR (BSP). BSP primers were designed according to the predicted $\mathrm{CpG}$ island in Rat-miR-210 
A
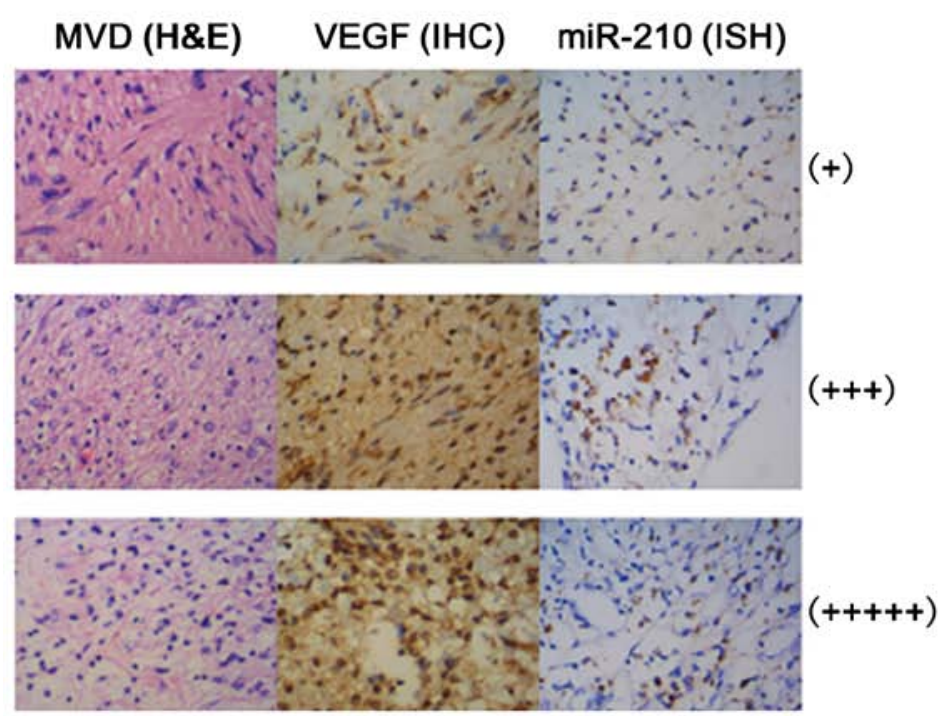

B

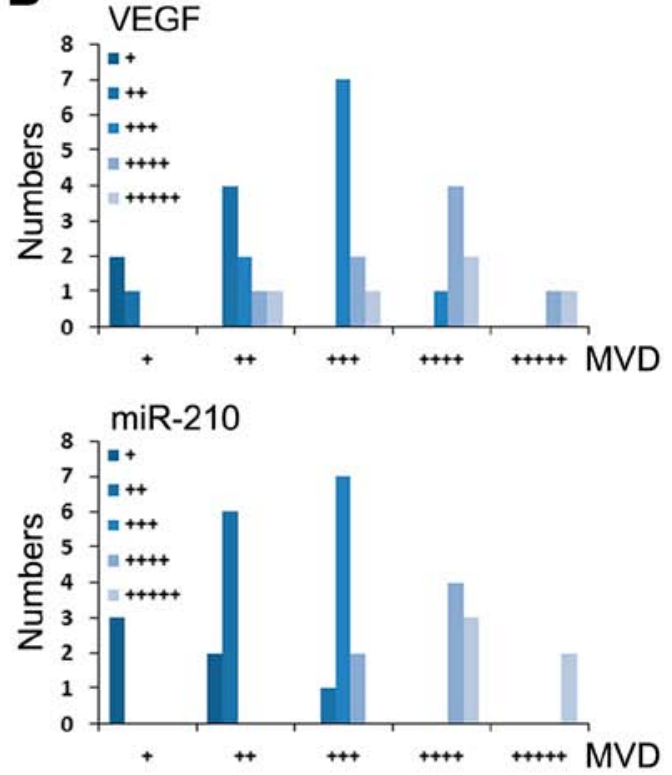

Figure 1. The expression of miR-210 and relationships with MVD and VEGF in schwannoma tissues. (A) Staining results under an optical microscope (x400): From the top to bottom, the figure shows the MVD test (H\&E staining), VEGF expression test (IHC) and miR-210 expression test (ISH) of 3 tumor tissue specimens with different microvascular densities. (B) Bar charts of Pearson's Chi-square test for MVD/VEGF and MVD/miR-210 of the tissue microarray, respectively; the distribution bars of MVD and VEGF (top), the distribution bars of MVD and miR-210 (bottom). (+) means positive expression level 1; (++) means positive expression level 2; by parity of reasoning, (+++++) means positive expression level 5.

promoter region: forward 5'-GGA AGG ATA TGT TTT GGA TTG TAT TAA-3', reverse 5'-TAA AAC CCA CCC TAC AAA ACT ACA AC-3', the PCR products were cloned into pGEM-T Easy vector, 15-20 clones were randomly selected for sequencing, and 10 correct clones were selected for methylation analysis.

Statistical analysis. The data are shown as the mean $\pm \mathrm{SE}$. Statistical analysis were performed using SPSS 11.0 software, Student's t-test was used to analyze the differences between two groups, with $95 \%$ confidence interval. Pearson's Chi-square test was used to analyze the relationships of microvascular density (MVD) vs. VEGF and MVD vs. miR-210, with $99 \%$ confidence interval. $\mathrm{P}<0.05$ was considered to indicate a statistically significant difference; and $\mathrm{P}<0.01$, highly significant.

\section{Results}

Expression of miR-210 and relationships with MVD and $V E G F$ in schwannoma tissues. As hypoxia is one of the key factors stimulating tumor vasculogenesis, we stained central neurilemmoma tissue microarray (with 30 cases of schwannoma tissues from different sources, 2 spots for each case) by H\&E, scored microvascular density (MVD), and then tested the expression of VEGF and miR-210 using the same chips by IHC and ISH, respectively (Fig. 1A). The bar chart of Pearson's Chi-squared test for MVD/VEGF and MVD/miR-210 relevance is shown in Fig. 1B, and the scores and $\mathrm{P}$-values as shown in Table I. VEGF positive stain became darker and miR-210 expression level increased with the tumor tissue MVD elevation, the MVD vs VEGF and MVD vs. miR-210 relevant levels had statistical significance at the level of confidence for interval of $99 \%(\mathrm{P}=0.005<0.01$, $\mathrm{P}=0.025<0.05)$.

Effects on miR-210/EFNA3 expression in rat schwann cells RT4-D6P2T under hypoxia. In order to explore the functions of miR-210 in schwannoma cells, we used RT4-D6P2T cells as a model for related research. First, to find whether hypoxia could affect miR-210/EFNA3 expression, the expression levels of miR-210 and EFNA3 were detected under hypoxia for $0,12,24$ and $48 \mathrm{~h}$ in RT4-D6P2T cells. As shown in Fig. 2, miR-210 expression increased slightly after $12 \mathrm{~h}$, increased by 4-6 times after $24 \mathrm{~h}$, and further increased after $48 \mathrm{~h}$, but not as much as $24 \mathrm{~h}$. While the expression trend of EFNA3 was opposite to that of miR-210. The expression of EFNA3 significantly decreased after $12 \mathrm{~h}$ hypoxic culture, and could hardly be detected after $24 \mathrm{~h}$ or $48 \mathrm{~h}$. This indicated that hypoxia induced the expression of miR-210 in RT4-D6P2T. EFNA3, one of the target genes of miR-210, is still negatively regulated by miR-210 during hypoxic culture and shows a reverse expression trend to $\mathrm{miR}-210$. This result was consistent with previous results (21). Thus, miR-210/EFNA 3 may be involved in schwannoma cell hypoxia. As expression of both miR-210 and EFNA3 changed significantly after $24 \mathrm{~h}$ in hypoxia, so we chose the hypoxic of $5 \% \mathrm{O}_{2}$ for $24 \mathrm{~h}$ as the following experimental conditions.

Effects on RT4-D6P2T cellular functions under hypoxia. As hypoxia changes miR-210/EFNA3 expression in schwannoma RT4-D6P2T cell line, we wanted to explore the effects of hypoxia on the functions of such cells, to find which function exerted hypoxia-induced miR-210/EFNA3 expression. We tested cell cycle, apoptosis and autophagy of the cells after $24 \mathrm{~h}$ hypoxic culture. Apoptosis increased greatly from $3 \%$ 
Table I. Bar chart of MVD and VEGF (Pearson's correlation=0.710)/miR-210 (Pearson's correlation=0.931).

\begin{tabular}{lcccccr}
\hline & \multicolumn{7}{c}{ VEGF } \\
\cline { 2 - 7 } MVD & $(+)$ & $(++)$ & $(+++)$ & $(++++)$ & $(+++++)$ & SUM \\
\hline$(+)$ & 2 & 1 & 0 & 0 & 0 & 3 \\
$(++)$ & 0 & 4 & 2 & 1 & 1 & 8 \\
$(+++)$ & 0 & 0 & 7 & 2 & 1 & 10 \\
$(++++)$ & 0 & 0 & 1 & 4 & 2 & 7 \\
$(+++++)$ & 0 & 0 & 0 & 1 & 1 & 2 \\
Total & 2 & 5 & 10 & 8 & 5 & 30 \\
\hline
\end{tabular}

$\operatorname{miR}-210$

\begin{tabular}{lcccccr} 
MVD & $(+)$ & $(++)$ & $(+++)$ & $(++++)$ & $(+++++)$ & SUM \\
\hline$(+)$ & 3 & 0 & 0 & 0 & 0 & 3 \\
$(++)$ & 2 & 6 & 0 & 0 & 0 & 8 \\
$(+++)$ & 0 & 1 & 7 & 2 & 0 & 10 \\
$(++++)$ & 0 & 0 & 0 & 4 & 3 & 7 \\
$(+++++)$ & 0 & 0 & 0 & 0 & 2 & 2 \\
Total & 5 & 7 & 7 & 6 & 5 & 30 \\
\hline
\end{tabular}

under normoxic conditions to $\sim 20 \%$ (Fig. 3A). The cell cycle arrested in G1 phase under hypoxic conditions (Fig. 3B).

Considering hypoxia could induce cell autophagy, we measured the cell autophagy under hypoxic culture by IF and western blotting. According to the results shown in Fig. 3C, during hypoxic culture, cellular morphology changed greatly, cells became larger and round in shape, which was associated with the cellular edema caused by too much water in cells due to hypoxia-induced sodium potassium pump function decrease. Besides, the IF staining results showed that the expression level of LC3BII increased, and the fluorescent signal around the cell nuclei significantly increased (Fig. 3C). According to Transwell experiment, although the quantity of the cells entering into Matrigel under the normoxic conditions was not significantly different with that in hypoxia, the cell morphology changed significantly, and under the hypoxic conditions, intercellular interval was wider, cells became narrower and fusiform shape (Fig. 3D). This may contribute to hypoxia-induced epithelial to mesenchymal transition phenomenon. According to the cell ultrastructure image by a transmission electron microscope (Fig. 3E), we found that during hypoxia, double membrane vacuole structures occurred in cytoplasm, and apoptotic bodies in cytoplasm significantly increased. Similarly, the results of western blotting on the autophagy related protein P62 and elf4E also showed that, after hypoxia P62 protein expression significantly decreased, while the expression level of the autophagy effector elf4E increased (Fig. 3F). Therefore, we concluded that hypoxia enhanced apoptosis and autophagy. In addition, according to Fig. 3F, HIF-1 $\alpha$ and VEGF expression increased after $24 \mathrm{~h}$ hypoxia; and considering miR-210

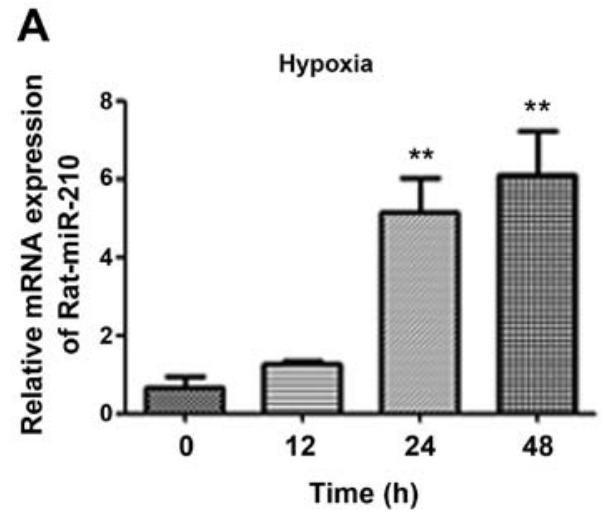

B

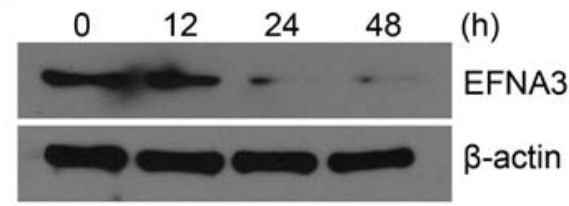

Figure 2. Effects on miR-210/EFNA3 expression by hypoxia. (A) miR-210 mRNA level in cells after different hypoxic culture periods using RT-qPCR. (B) The protein expression of EFNA3 by western blotting. ${ }^{* *} \mathrm{P}<0.01$.

expression increased and EFNA3 expression decreased during hypoxia together (Fig. 2A), we speculated that during hypoxia, the miR-210 enhancement of schwannoma cells was induced by HIF-1 $\alpha$, and miR-210 regulated vascularization by targeting EFNA3.

Mechanism of normoxia/hypoxia-induced miR-210 regulation. To investigate whether miR-210 upregulation during hypoxia is induced by HIF-1 $\alpha$, we carried out HIF-1 $\alpha$ silencing by HIF-1 $\alpha$-shRNA, and then placed the HIF-1 $\alpha$ loss-of-funciton cells under normoxic/hypoxic conditions, respectively for $24 \mathrm{~h}$. Hypoxia can induce miR-210 upregulation, but after HIF-1 $\alpha$ interference, miR-210 expression level decreased significantly (Fig. 4A). This indicated that miR-210 expression did relate to $\mathrm{HIF}-1 \alpha$ expression, and HIF-1 $\alpha$ may positively regulate miR-210 expression.

We further studied the mechanism of how hypoxia induced miR-210 upregulation. We predicted that there were abundant $\mathrm{CpG}$ islands in the promoter region of rat miR-210 (Fig. 4B) by the online software Lilab (http://www. urogene.org/cgi-bin/methprimer/methprimer.cgi), $\mathrm{CpG}$ Island Searcher (http://cpgislands.usc.edu/), and EMBOSS explorer (http://emboss.bioinformatics.nl/cgi-bin/emboss/ cpgplot). Moreover, the early methylation-specific PCR (MSP) preliminary experiment results showed that under hypoxic conditions, the miR-210 promoter region methylation level in cells decreased significantly compared with that under normoxic conditions (data not shown). We speculated that during hypoxia, demethylation occurred in the miR-210 promoter region, which enabled more hypoxia-induced HIF-1 $\alpha$ to combine with HRE in the miR-210 promoter region and activated miR-210 translation. So, we first tested the methylation islands of the miR-210 promoter region by Bisulfite sequencing PCR (BSP) under hypoxia, and then assessed whether HRE combined with HIF-1 $\alpha$ was included in these demethylated $\mathrm{CpG}$ islands. The results showed that 
A

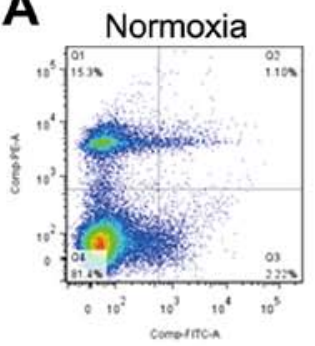

B

B Normoxia

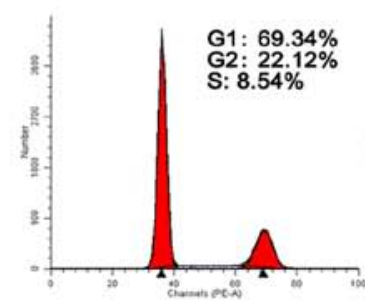

D

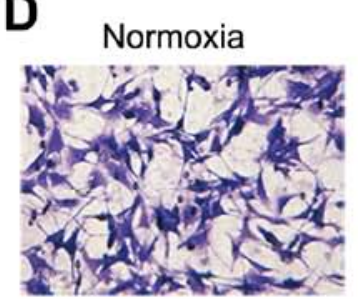

E

a

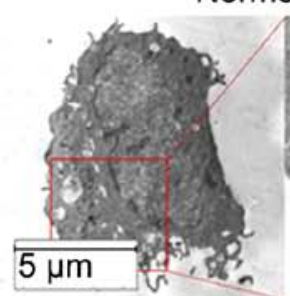

Hypoxia

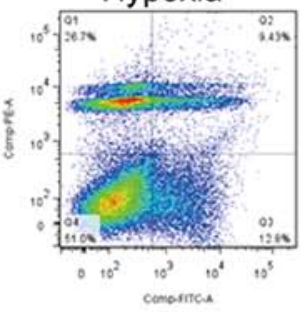

Hypoxia

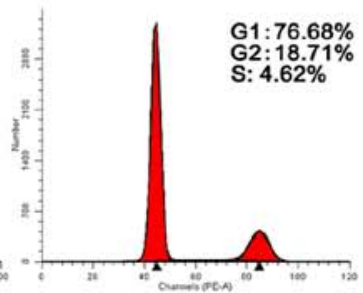

Hypoxia

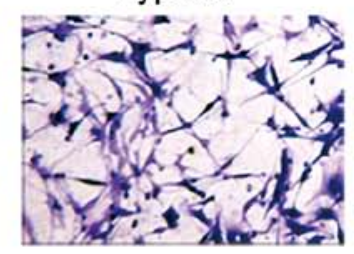

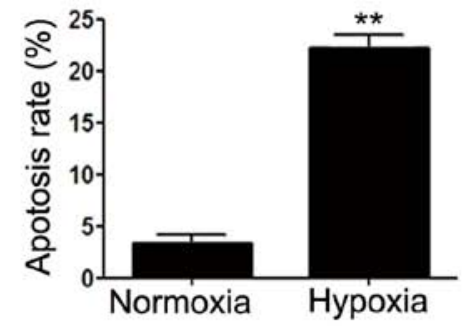

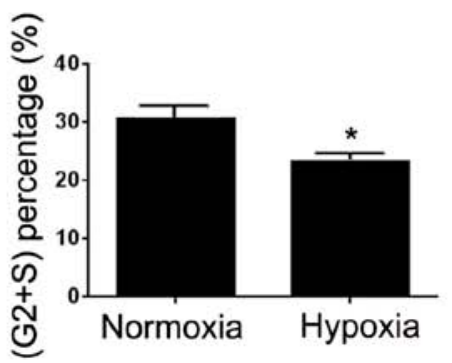

C

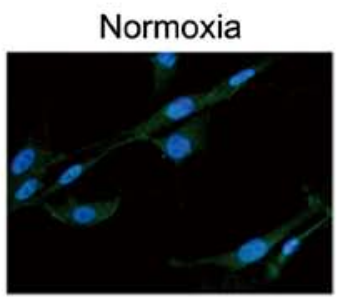

Hypoxia

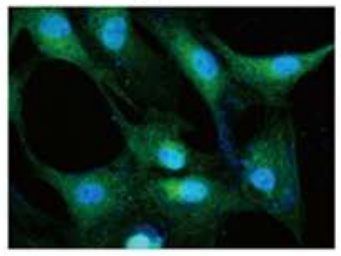

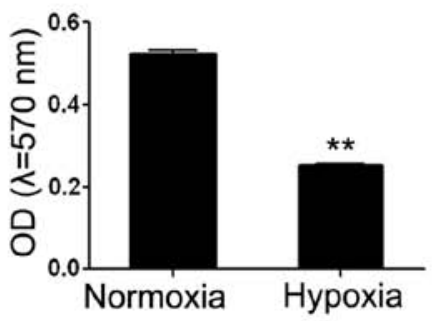

b
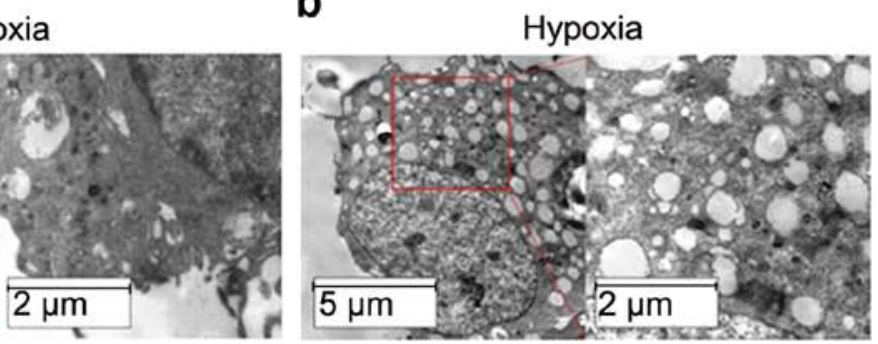

$\mathbf{F}$
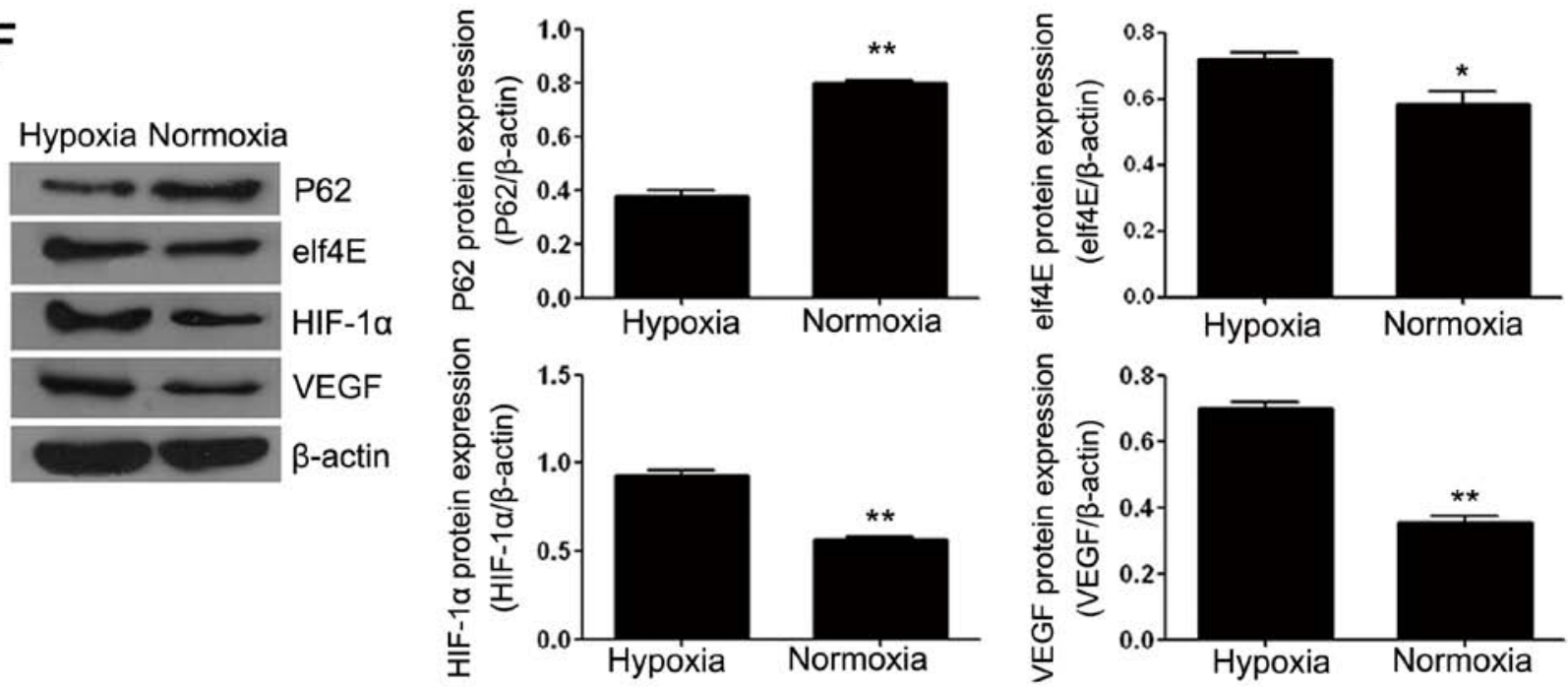

Figure 3. Cell apoptosis, cycle, autophagy, invasion and related protein expression under normoxic or hypoxic conditions. (A) Flow cytometry on apoptosis rate under normoxic and hypoxic conditions after $24 \mathrm{~h}$. (B) The flow cytometry on cell cycle under normoxic and hypoxic conditions, with the proportion of $\mathrm{S}+\mathrm{G} 2$ phase cells as the basis for judging cell cycle progress. (C) Immunofluorescent image (x400) showing the intracellular LC3B II/I protein expression. (D) Cell invasion was measured using Transwell assay (x200). (E) Cell ultrastructure changes under a transmission electron microscope; (a) cellular structure under normoxic culture; (b) cellular structure under hypoxic culture. (F) Western blotting on protein expression of HIF-1 $\alpha$, VEGF and the autophagy related factor P62 and elf4E. ${ }^{*} \mathrm{P}<0.05,{ }^{* *} \mathrm{P}<0.01$. 
A

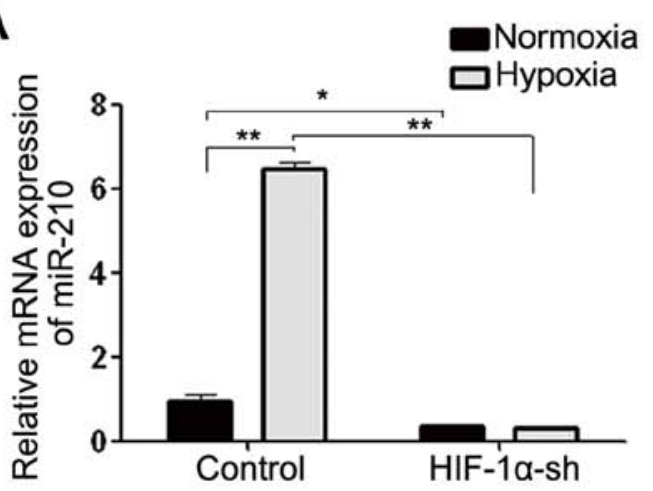

B
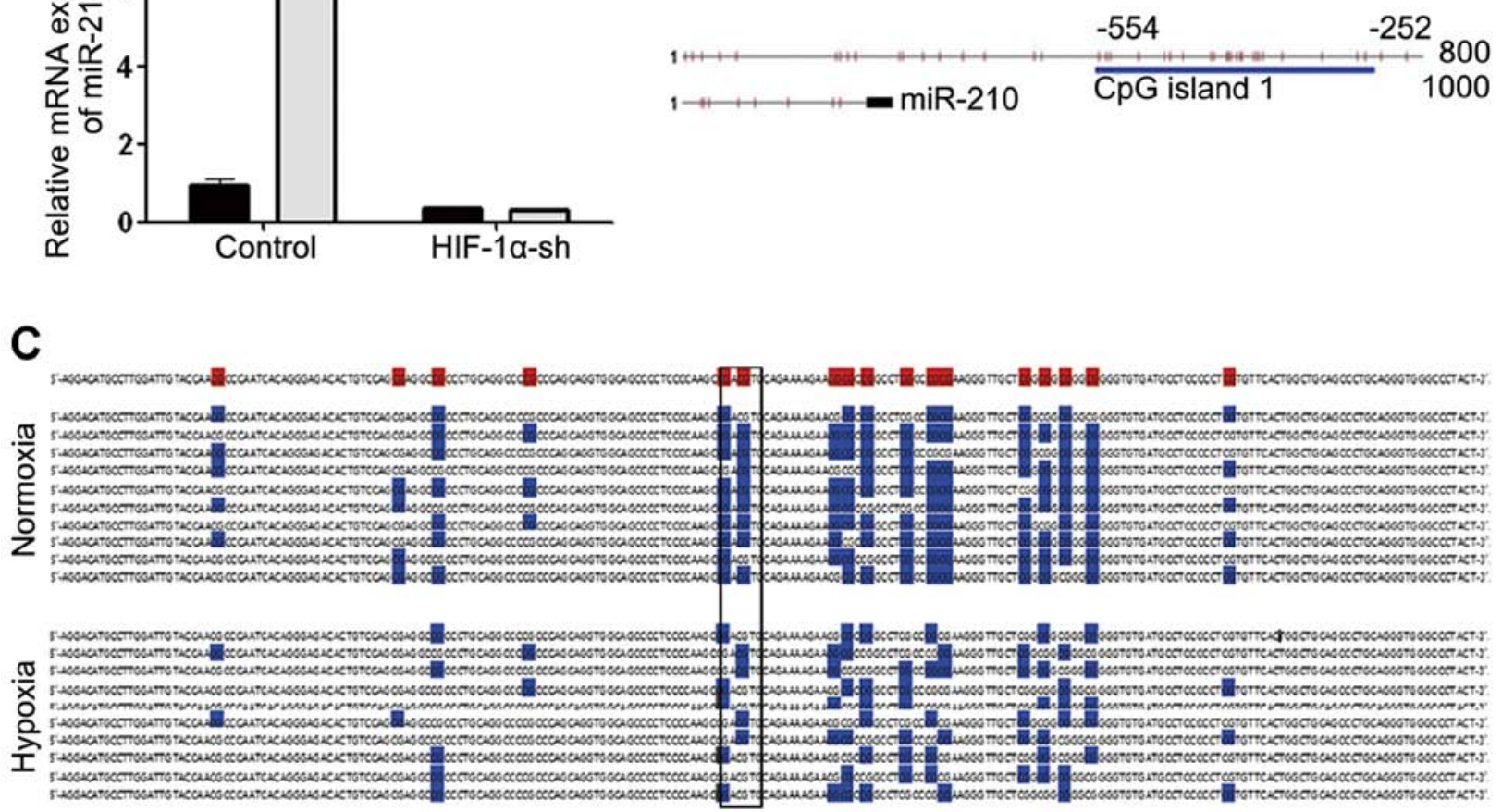

Figure 4. Mechanism of hypoxia-induced miR-210 expression. (A) miR-210 mRNA expression levels in each group of cells by RT-qPCR. (B) The Rat-miR-210 promoter region $\mathrm{CpG}$ islands predicted by $\mathrm{CpG}$ island searcher. (C) The miR-210 promoter region methylation levels of RT4-D6P2T line after normoxic and hypoxic culture. The 17 potential $\mathrm{CpG}$ loci are highlighted by red bars, and the $\mathrm{CpG}$ loci involving methylation as tested by BSP were highlighted by blue bars, and the sequences in the black boxes represent HIF-1 $\alpha$ binding sites. BSP sequencing was repeated 15-20 times under both normoxic and hypoxic conditions, and the correct sequencing results of 10 tests are shown. ${ }^{*} \mathrm{P}<0.05 .{ }^{* * *} \mathrm{P}<0.01$.

there were $17 \mathrm{CpG}$ locuses in the upstream -252 to $-554 \mathrm{bp}$ region of the miR-210 coding region, and under the normoxic conditions, the methylation level of this $\mathrm{CpG}$ islands segment was $\sim 72.35 \%$, while after $24 \mathrm{~h}$ hypoxic culture, the methylation level dcreased significantly to $\sim 44.70 \%$ (Fig. 4C).

miR-210 plays important roles in RT4-D6P2T schwannoma cell line under normoxic/hypoxic conditions. In order to clarify the function of miR-210 on schwannoma cells during hypoxia, we built miR-210 knockdown cell model and then placed the model cells into normoxic/hypoxic conditions for 24 h, respectively. As shown in Fig. 5A, compared with the negative control group (anti-scramble), the proportion of the cells in the $\mathrm{S}+\mathrm{G} 2$ phase significantly reduced in anti-miR-210 group, but the quantity difference of the $\mathrm{S}+\mathrm{G} 2$ phase between the normoxia and the hypoxia group does not significant. The cell apoptosis rates increased significantly in anti-miR-210 group in both the normoxia and the hypoxia group (Fig. 5B). LC3B II fluorescence intensities of anti-miR-210 group are lower than those of the anti-scramble group, and higher (under hypoxic conditions) than those of the cells cultured under normoxic conditions (Fig. 5C).

According to the transmission electron microscope scanning results (Fig. 5E), under normoxic conditions, cellular matrix density was uniform and with few autolysosomes; after hypoxic culture, double membrane vacuole structures occurred in cellular matrix, mitochondria breakage was more serious, some damaged mitochondria were covered by dual-membranes, and autophagy-induced cavitation in cells of the miR-210 group were much less than those of the antiscramble group. This indicated that miR-210 could mediate cell autophagy. Western blot results on the autophagy related factors (P62 and elf4E) were consistent with the aforementioned results (Fig. 5F). The expression level of HIF- $1 \alpha$ and VEGF protein also decreased when the miR-210 was knocked down, while EFNA3 expression increased (Fig. 5F). This indicated that miR-210/EFNA3 was related with the HIF-1 $\alpha /$ VEGF-mediated neovascularization paths.

\section{Discussion}

According to this study, we found that under hypoxia, HIF-1 $\alpha$, miR-210 and VEGF expression levels in RT4-D6P2T cells were significantly higher, EFNA3 expression was significantly lower, apoptosis higher, autophagy occurred, and cell cycle was arrested. In addition, intracellular miR-210 expression upregulation under hypoxia was found to be associated with its transcription start region $\mathrm{CpG}$ island demethylation for the first time.

It seems hypoxia can induce tumor cell apoptosis and necrosis, and thus can inhibit excessive proliferation, but finally it may do more harm than good. Because after apoptosis, necrotic cells release inflammation signals to the tissue microenvironment around them and recruit inflammatory cells 



Figure 5. miR-210 plays an important role in cell function under normoxic/hypoxic conditions. (A) The flow cytometry result on single stained cell cycle, statistical result of (S+G2)\% is shown in the figure (top of D). (B) Flow cytometry results on double-stained cell apoptosis, statistical result of (R3+R5) \% is shown in the figure (bottom of D). (C) LC3BII/I protein fluorescence intensity under $\mathrm{x} 400$ microscope. (E) Cell ultrastructure under transmission electron microscope, of which (a) the negative control group cells under normoxic conditions, (b) the groups without miR-210 function under normoxic conditions, (c) the negative control group cells after $24 \mathrm{~h}$ hypoxic culture, (d) the groups without miR-210 function after $24 \mathrm{~h}$ hypoxic culture. (F) Western blot results on intracellular EFNA3, P62, elf4E, p-AKT, HIF-1 $\alpha$, VEGF protein expression change grey-scale map and statistical chart of each group. ${ }^{*} \mathrm{P}<0.05$. ** $\mathrm{P}<0.01$.

of the immune system $(29,30)$, which can promote vasculogenesis, cancer cell proliferation and infiltration, can also directly activate the regulatory factors for adjacent viable cell proliferation, such as IL-1 $\alpha$ and finally promote tumor growth (30). 
Autophagy is an important cell physiological reaction similar to apoptosis. Normal cells are at a low level, but autophagy can be strongly activated under some cellular stress states, of which the most significant stress state is nutrition deficiency (31). The nutrition deficiency can induce cellular autophagy level increase, and thus is able to resist rather than enhance environmental stress destruction process of tumor cells (32). In addition, in an adverse environment, cells can shrink to a reversible dormant state via autophagy contraction, which may enable some advanced tumors to survive and finally relapse (33).

Our invasion experiment results showed that hypoxia did not increase the invasion of cells, but caused fibrinoid changes of cells, enabled them to grow filopodium, whose main functions consisted of movement, adhesion, support, nutrition absorption and phagocytosis, and thus increased invasion capabilities of tumor cells. Thus, we can see that although hypoxia has an unfavorable influence on the growth of the tumor, it is still an important factor that can induce tumor progress acceleration.

Since miRNAs can maintain pluripotency of cancer stem cells/progenitor cells, and may become a molecule therapeutic target or biomarker of cancers, they have attracted more and more attentions from scholars (34). However, the mechanism of how tumor cells regulate miRNA expression has not been clearly revealed. Bioinformatics analysis results showed that there were $\mathrm{CpG}$ islands in the transcription start site (TSS) regions of 129 of 721 human miR genes (accounting for 18\%) in miRBase (http://www.mirbase.org/) (34). Some related references (35-40) also showed that $\mathrm{CpG}$ island methylation or high methylation level in TSS regions of miRNAs was thought to be inhibited by hysterogenic (epigenetic) transcription. However, the relationship between miR methylation and miR expression has not been completely revealed, and the previous evidence on such a relationship is not sufficient.

We discovered that hypoxia could induce miR-210 promoter region demethylation. Huang et al (17) revealed the locus where miR-210 promoter region could combine with HIF-1 $\alpha$ as 5'-GAC GTG-3', upstream -429 to -423 bp of miR-210 coding region. Our BSP test results showed that there were 2 methylation loci in this region which showed high methylation levels under normoxic conditions, while low methylation levels under hypoxic conditions. This probably enhanced the combining capacity of HIF-1 $\alpha$ with HRE in miR-210 TSS region, and further enhanced miR-210 transcriptional activity.

Moreover, in the oxygen-independent regulating system, miR-210 can induce HIF-1 $\alpha$ expression level increase via inhibiting prolyl hydroxylases which can degrade HIF-1 $\alpha$ (9). The repression of succinate dehydrogenase complex subunit D (a HIF-1 $\alpha$ inhibitor) by miR-210 can also contribute to the maintence of HIF- $1 \alpha$ activity, then further promote miR-210 transcription and enhance the feedback regulation (41). Such hypoxia-induced HIF-1 $\alpha / \mathrm{miR}-210$ feedback regulation mechanism directly promotes schwannoma cell survival, autophagy protection, and tumor cell changes from epithelial type to mesenchymal type during hypoxia, and consequently increases possibility of malignancy transformation. Therefore, miR-210 has the potential to become an index for testing internal hypoxia of schwannoma tissues, and can also be taken as an index for judging whether the tumor is of malignancy transformation trend.

Similarly, we silenced miR-210 of RT4-D6P2T cells to prove our conclusion, and compared the miR-210 functional role under normoxic/hypoxic conditions. As anticipated, the results showed miR-210 participated in many biological functions, such as regulating RT4-D6P2T cell anti-apoptosis, autophagy protection and invasion ability, and promoted tumor cell cycle. Thus, developing a specific miR-210 inhibitor will be significant for neurilemmoma treatment.

miR-210 can be taken as a biomarker for diagnosing neurilemmoma hypoxia. Hypoxia-induced HIF-1 $\alpha / \mathrm{miR}-210$ expression upregulation can negatively regulate EFNA3 protein expression level, and enhance tumor neovascularization. Hypoxia-induced schwannoma cell apoptosis and autophagy level increase as well as invasion potential increase can be reversed by anti-miR-210 treatment, and miR-210 is a potential neurilemmoma therapeutic target.

\section{Acknowledgements}

This study was supported by the New Xiangya Talent Project of The Third Xiangya Hospital of Central South University (grant no. JY201516).

\section{References}

1. Masliah-Planchon J, Pasmant E, Luscan A, Laurendeau I, Ortonne N, Hivelin M, Varin J, Valeyrie-Allanore L, Dumaine V, Lantieri L, et al: MicroRNAome profiling in benign and malignant neurofibromatosis type 1-associated nerve sheath tumors: Evidences of PTEN pathway alterations in early NF1 tumorigenesis. BMC Genomics 14: 473, 2013.

2. Harris AL: Hypoxia - a key regulatory factor in tumour growth. Nat Rev Cancer 2: 38-47, 2002.

3. Camps C, Saini HK, Mole DR, Choudhry H, Reczko M, GuerraAssunção JA, Tian YM, Buffa FM, Harris AL, Hatzigeorgiou AG, et al: Integrated analysis of microRNA and mRNA expression and association with HIF binding reveals the complexity of microRNA expression regulation under hypoxia. Mol Cancer 13: 28, 2014.

4. Semenza GL: Hypoxia-inducible factor 1: Master regulator of $\mathrm{O}_{2}$ homeostasis. Curr Opin Genet Dev 8: 588-594, 1998.

5. Ben-Yosef Y, Miller A, Shapiro S and Lahat N: Hypoxia of endothelial cells leads to MMP-2-dependent survival and death. Am J Physiol Cell Physiol 289: C1321-C1331, 2005.

6. Hanahan D and Weinberg RA: Hallmarks of cancer: The next generation. Cell 144: 646-674, 2011.

7. Kitano H: Cancer as a robust system: Implications for anticancer therapy. Nat Rev Cancer 4: 227-235, 2004.

8. Melillo G and Semenza GL: Meeting report: Exploiting the tumor microenvironment for therapeutics. Cancer Res 66: 4558-4560, 2006.

9. Nallamshetty S, Chan SY and Loscalzo J: Hypoxia: A master regulator of microRNA biogenesis and activity. Free Radic Biol Med 64: 20-30, 2013.

10. Valencia-Sanchez MA, Liu J, Hannon GJ and Parker R: Control of translation and mRNA degradation by miRNAs and siRNAs. Genes Dev 20: 515-524, 2006.

11. Gee HE, Ivan C, Calin GA and Ivan M: HypoxamiRs and cancer: From biology to targeted therapy. Antioxid Redox Signal 21: 1220-1238, 2014. 10.1089/ars.2013.5639.

12. Chen KC, Liao YC, Wang JY, Lin YC, Chen CH and Juo SH: Oxidized low-density lipoprotein is a common risk factor for cardiovascular diseases and gastroenterological cancers via epigenomical regulation of microRNA-210. Oncotarget 6 : 24105-24118, 2015 .

13. Yang Y, Qu A, Liu J, Wang R, Liu Y, Li G, Duan W, Fang Q, Jiang $\mathrm{X}$, Wang L, et al: Serum miR-210 contributes to tumor detection, stage prediction and dynamic surveillance in patients with bladder cancer. PLoS One 10: e0135168, 2015. 
14. Bertoli G, Cava C and Castiglioni I: MicroRNAs: New biomarkers for diagnosis, prognosis, therapy prediction and therapeutic tools for breast cancer. Theranostics 5: 1122-1143, 2015.

15. Humeau M, Vignolle-Vidoni A, Sicard F, Martins F, Bournet B, Buscail L, Torrisani J and Cordelier P: Salivary MicroRNA in pancreatic cancer patients. PLoS One 10: e0130996, 2015.

16. Dang K and Myers KA: The role of hypoxia-induced miR-210 in cancer progression. Int J Mol Sci 16: 6353-6372, 2015.

17. Huang X, Ding L, Bennewith KL, Tong RT, Welford SM, Ang KK, Story M, Le QT and Giaccia AJ: Hypoxia-inducible mir-210 regulates normoxic gene expression involved in tumor initiation. Mol Cell 35: 856-867, 2009.

18. Huang X, Le QT and Giaccia AJ: MiR-210 - micromanager of the hypoxia pathway. Trends Mol Med 16: 230-237, 2010.

19. Ying Q, Liang L, Guo W, Zha R, Tian Q, Huang S, Yao J, Ding J, Bao M, Ge C, et al: Hypoxia-inducible microRNA-210 augments the metastatic potential of tumor cells by targeting vacuole membrane protein 1 in hepatocellular carcinoma. Hepatology 54 2064-2075, 2011.

20. Hwang HW, Baxter LL, Loftus SK, Cronin JC, Trivedi NS, Borate B and Pavan WJ: Distinct microRNA expression signatures are associated with melanoma subtypes and are regulated by HIF1A. Pigment Cell Melanoma Res 27: 777-787, 2014.

21. Fasanaro P, D'Alessandra Y, Di Stefano V, Melchionna R, Romani S, Pompilio G, Capogrossi MC and Martelli F: MicroRNA-210 modulates endothelial cell response to hypoxia and inhibits the receptor tyrosine kinase ligand Ephrin-A3. J Biol Chem 283: 15878-15883, 2008.

22. Miao H and Wang B: EphA receptor signaling - complexity and emerging themes. Semin Cell Dev Biol 23: 16-25, 2012

23. Larson J, Schomberg S, Schroeder W and Carpenter TC Endothelial EphA receptor stimulation increases lung vascular permeability. Am J Physiol Lung Cell Mol Physiol 295: L431-L439, 2008.

24. Genander M, Halford MM, Xu NJ, Eriksson M, Yu Z, Qiu Z, Martling A, Greicius G, Thakar S, Catchpole T, et al: Dissociation of EphB2 signaling pathways mediating progenitor cell proliferation and tumor suppression. Cell 139: 679-692, 2009.

25. Uchiyama S, Saeki N and Ogawa K: Aberrant EphB/ephrin-B expression in experimental gastric lesions and tumor cells. World J Gastroenterol 21: 453-464, 2015.

26. Wang Z, Liu Z, Liu B, Liu G and Wu S: Dissecting the roles of Ephrin-A3 in malignant peripheral nerve sheath tumor by TALENs. Oncol Rep 34: 391-398, 2015.

27. Wang Z, Yin B, Wang B, Ma Z, Liu W and Lv G: MicroRNA-210 promotes proliferation and invasion of peripheral nerve sheath tumor cells targeting EFNA3. Oncol Res 21: 145-154, 2013.
28. Mao L, Zhou Q, Zhou S, Wilbur RR and Li X: Roles of apolipoprotein $\mathrm{E}$ (ApoE) and inducible nitric oxide synthase (iNOS) in inflammation and apoptosis in preeclampsia pathogenesis and progression. PLoS One 8: e58168, 2013.

29. Galluzzi L and Kroemer G: Necroptosis: A specialized pathway of programmed necrosis. Cell 135: 1161-1163, 2008.

30. Grivennikov SI, Greten FR and Karin M: Immunity, inflammation, and cancer. Cell 140: 883-899, 2010.

31. Levine B and Kroemer G: Autophagy in the pathogenesis of disease. Cell 132: 27-42, 2008.

32. Amaravadi RK and Thompson CB: The roles of therapy-induced autophagy and necrosis in cancer treatment. Clin Cancer Res 13 7271-7279, 2007

33. Lu Z, Luo RZ, Lu Y, Zhang X, Yu Q, Khare S, Kondo S, Kondo Y, Yu Y, Mills GB, et al: The tumor suppressor gene ARHI regulates autophagy and tumor dormancy in human ovarian cancer cells. J Clin Invest 118: 3917-3929, 2008.

34. Du Y, Liu Z, Gu L, Zhou J, Zhu BD, Ji J and Deng D: Characterization of human gastric carcinoma-related methylation of $9 \mathrm{miR} \mathrm{CpG}$ islands and repression of their expressions in vitro and in vivo. BMC Cancer 12: 249, 2012.

35. Furuta M, Kozaki KI, Tanaka S, Arii S, Imoto I and Inazawa J: miR-124 and miR-203 are epigenetically silenced tumor-suppressive microRNAs in hepatocellular carcinoma. Carcinogenesis 31: 766-776, 2010.

36. Tsuruta T, Kozaki K, Uesugi A, Furuta M, Hirasawa A, Imoto I, Susumu N, Aoki D and Inazawa J: miR-152 is a tumor suppressor microRNA that is silenced by DNA hypermethylation in endometrial cancer. Cancer Res 71: 6450-6462, 2011.

37. Tsai KW, Wu CW, Hu LY, Li SC, Liao YL, Lai CH, Kao HW, Fang WL, Huang KH, Chan WC, et al: Epigenetic regulation of miR-34b and miR-129 expression in gastric cancer. Int J Cancer 129: 2600-2610, 2011.

38. Hildebrandt MA, Gu J, Lin J, Ye Y, Tan W, Tamboli P, Wood CG and Wu X: Hsa-miR-9 methylation status is associated with cancer development and metastatic recurrence in patients with clear cell renal cell carcinoma. Oncogene 29: 5724-5728, 2010.

39. Hashimoto Y, Akiyama Y, Otsubo T, Shimada S and Yuasa Y: Involvement of epigenetically silenced microRNA-181c in gastric carcinogenesis. Carcinogenesis 31: 777-784, 2010.

40. Yang C, Cai J, Wang Q, Tang H, Cao J, Wu L and Wang Z: Epigenetic silencing of miR-130b in ovarian cancer promotes the development of multidrug resistance by targeting colonystimulating factor 1 . Gynecol Oncol 124: 325-334, 2012.

41. Gorospe M, Tominaga K, Wu X, Fähling M and Ivan M: Posttranscriptional control of the hypoxic response by RNA-binding proteins and microRNAs. Front Mol Neurosci 4: 7, 2011. 